\title{
Natural Course of Children With Dysplastic and Hypoplastic Kidney
}

\author{
Nida Dincel ${ }^{\mathrm{a}, \mathrm{d}}$, Hasan Bicer ${ }^{\mathrm{b}}$, Zubeyir Hasan Gun ${ }^{\mathrm{c}}$, Sevgi Mir ${ }^{\mathrm{a}}$
}

\begin{abstract}
Background: The objective of this study was to report the outcomes of children with dysplastic kidney and identify the variables that are independent predictors on the renal involution.

Methods: We reviewed the clinical, radiological, and nuclear medicine findings of 48 children ( 30 boys and 18 girls) with dysplastic or hypodisplastic kidney who were diagnosed and treated in our center between 1999 and 2012.
\end{abstract}

Results: A total of 25 cases (52\%) were diagnosed prenatally and $23(48 \%)$ postnatally. Twenty-six cases $(54.1 \%)$ had pathologic findings in the contralateral kidney. During the follow-up, 21 patients $(43.7 \%)$ showed completely involution within 2 years and $16(33.3 \%)$ showed a partial involution. The univariate analysis showed that no variables were associated with complete involution. On laboratory evaluation, the mean creatinine level was $1.66 \pm 2.1$ $\mathrm{mg} / \mathrm{dL}$ and the mean estimated glomerular filtration rate (eGFR) was $75.8 \pm 55.6 \mathrm{~mL} / \mathrm{min}$. Eleven patients $(22.9 \%)$ had an eGFR $<30 \mathrm{~mL} / \mathrm{min} / 1.73 \mathrm{~m}^{2}$, of which 7 had contralateral renal dysplasia and 4 had contralateral reflux nephropathy. Nevertheless, at last follow-up, 18 children were in end-stage renal failure $(37.5 \%)$. Proteinuria was noted in 11(22.9\%) patients, of whom all had nephrotic range proteinuria. Fifteen (31.2\%) cases had hypertension at diagnosis but no children developed new onset hypertension during the follow-up. Furthermore, no neoplastic degeneration was documented in any case.

Conclusions: The prognosis of dysplastic kidneys depends on the

Manuscript accepted for publication October 14, 2013

${ }^{\mathrm{a} D e p a r t m e n t ~ o f ~ P e d i a t r i c ~ N e p h r o l o g y, ~ F a c u l t y ~ o f ~ M e d i c i n e, ~ E g e ~}$ University, Izmir, Turkey

${ }^{b}$ Faculty of Medicine, Ege University, Izmir, Turkey

${ }^{c}$ Faculty of Medicine, Emory University, Atlanta, USA

${ }^{\mathrm{d}}$ Corresponding author: Nida Dincel, Department of Pediatric

Nephrology, Faculty of Medicine, Ege University, Izmir, Turkey.

Email: nida_dincel@yahoo.com

doi: http://dx.doi.org/10.4021/wjnu122w function of the contralateral kidney, so these patients should be monitored regularly assessing the dysplastic and contralateral kidney, blood pressure, urinalysis, and renal function.

Keywords: Chronic renal failure; Dysplastic kidney; Follow-up; Hypoplastic kidney; Pediatrics

\section{Introduction}

Abnormal maturation of kidneys leads to a wide spectrum of congenital urinary tract anomalies including renal dysplasia and hypoplasia [1]. Hypoplastic and dysplastic kidneys are common malformations affecting up to 1 in 1,000 of the general population. Renal hypoplasia is defined as a small sized kidney, which contains intact nephrons that are reduced in number, whereas a dysplastic kidney contains disorganized renal tissue, involving undifferentiated epithelium, primitive ducts, and fibromuscular connective tissue [2].

These congenital anomalies are the most frequent cause of chronic renal failure (CRF) in childhood, accounting for $25 \%$ of children requiring renal replacement therapy [3-5]. However, the progression towards end-stage renal disease is highly variable between the patients [3]. Furthermore. the natural history and long-term renal prognosis of these diseases are poorly defined, thus making it difficult to predict renal outcomes of any individual patient with dysplastic or hypoplastic kidney [5-8].

In the present study, we analyzed the natural course of renal function in a single-center cohort of children with dysplastic or hypodisplastic kidney and identified variables that are independent predictors on the involution of kidney.

\section{Patients and Methods}

We reviewed the clinical, radiological, and nuclear medicine findings of 48 children (30 boys and 18 girls) with dysplastic or hypodisplastic kidney who were diagnosed and treated in our center between 1999 and 2012. All patients were diagnosed by prenatal and/or postnatal ultrasound (US), voiding 
Table 1. Demographic Characteristics of the Patients

\begin{tabular}{ll}
\hline No. of patients & 48 \\
Male/female & $30 / 18$ \\
Prenatal diagnosis & $25(52 \%)$ \\
Affected kidney & \\
$\quad$ Right & $30(62.5 \%)$ \\
Left & $11(22.9 \%)$ \\
Bilateral & $7(14.5 \%)$ \\
Compensatory hypertrophy & $11(22.9 \%)$ \\
Associated abnormalities & $17(35.4 \%)$ \\
Hydronephrosis in contralateral kidney & $6(12.5 \%)$ \\
Vesicoureteral reflux & $1(2 \%)$ \\
Ureteropelvic junction obstruction & $1(2 \%)$ \\
Crossed renal ectopy & $1(2 \%)$ \\
Posterior urethral valve & $3(6.2 \%)$ \\
Neurogenic bladder & $2(4.1 \%)$ \\
Cardiomyopathy & $1(2 \%)$ \\
Down syndrome & $1(2 \%)$ \\
\hline Cryptorchidism & $1(2 \%)$ \\
\hline
\end{tabular}

cystourethrography (VCUG) and/or renal scan. Follow-up period was longer than 60 months in all cases. Follow-up US examinations were performed at 6-month intervals during the first 2 years of life and yearly thereafter. Compensatory hypertrophy of the contrlateral kidney was defined as a renal lentgh greater than +2 standard deviations of the mean value of normal kidneys [9]. Increased renal echogenicity was defined as echogenicity identical to or more than liver echogenicity [10].

Prophylactic antibiotics were administered when vesicoureteral reflux (VUR) was detected. Renal scintigraphy was performed with technetium-99m, dimercaptosuccinic acid (99mTc-DMSA) to confirm diagnosis and evaluate renal scarring in the contralateral kidney. Blood pressure was measured in all children by an oscillometric device and treated with antihypertensive drugs if more than three values of blood pressure were $>90$ th percentile for age and sex, according to the Task Force Study [11]. Additionally serum urea and creatinine measurements, glomerular filtration rate (GFR), urinalysis and urine culture were performed at baseline and periodically repeated thereafter. The GFR was calculated using the formula of Schwartz et al [12].

\section{Statistical analyses}

SPSS version 15.0 statistics program was used for statistical calculations. Results were shown as means and standard deviations (SD), or if not normally distributed, median and ranges. Chi-square, Fisher's exact, and Mann-Whitney U tests were performed for statistical analysis. A P value $<0.05$ was defined as significant.

\section{Results}

We identified 48 patients (30 boys and 18 girls) followed 


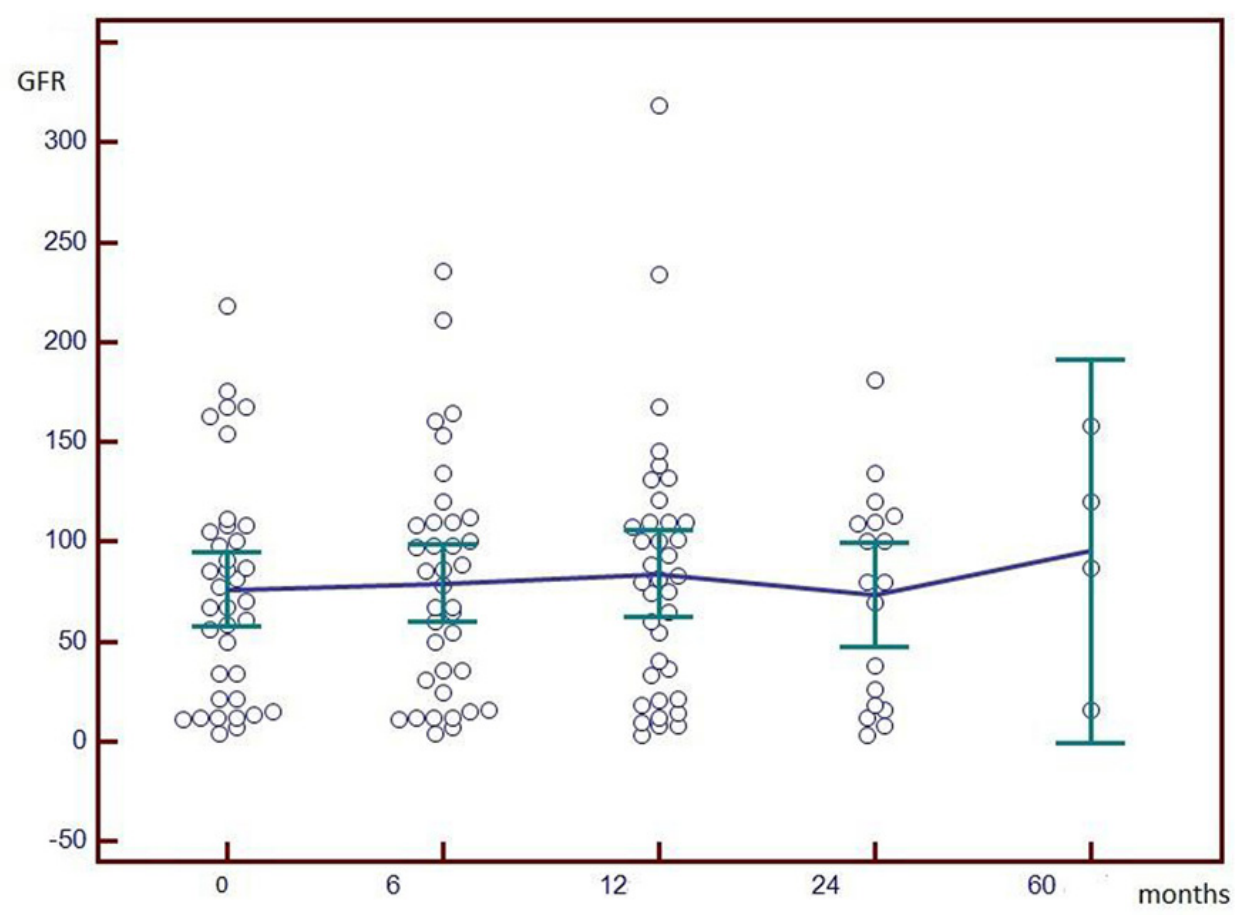

Figure 1. Involution rate of dysplatic kidneys.

with US after initial evaluation with prenatal and/or postnatal US, VCUG, and renal scintigraphy. A total of 25 cases $(52 \%)$ were diagnosed prenatally by ultrasound and 23 $(48 \%)$ postnatally. Affected kidney was on the right side in 30 patients $(62.5 \%)$, on the left side in $11(22.9 \%)$, and bilateral in $7(14.5 \%)$. The demographic characteristics of the patients are summarized in Table 1.

Of the 48 cases, 26 (54.1\%) had pathologic findings in the contralateral kidney on US, consisting of hydronephrosis in 17 , renal dysplasia in 7 , ureteropelvic junction obstruction in 1 , and crossed renal ectopy in 1 . The reason of hydronephrosis was VUR in 6 patients, neurogenic bladder in 3,

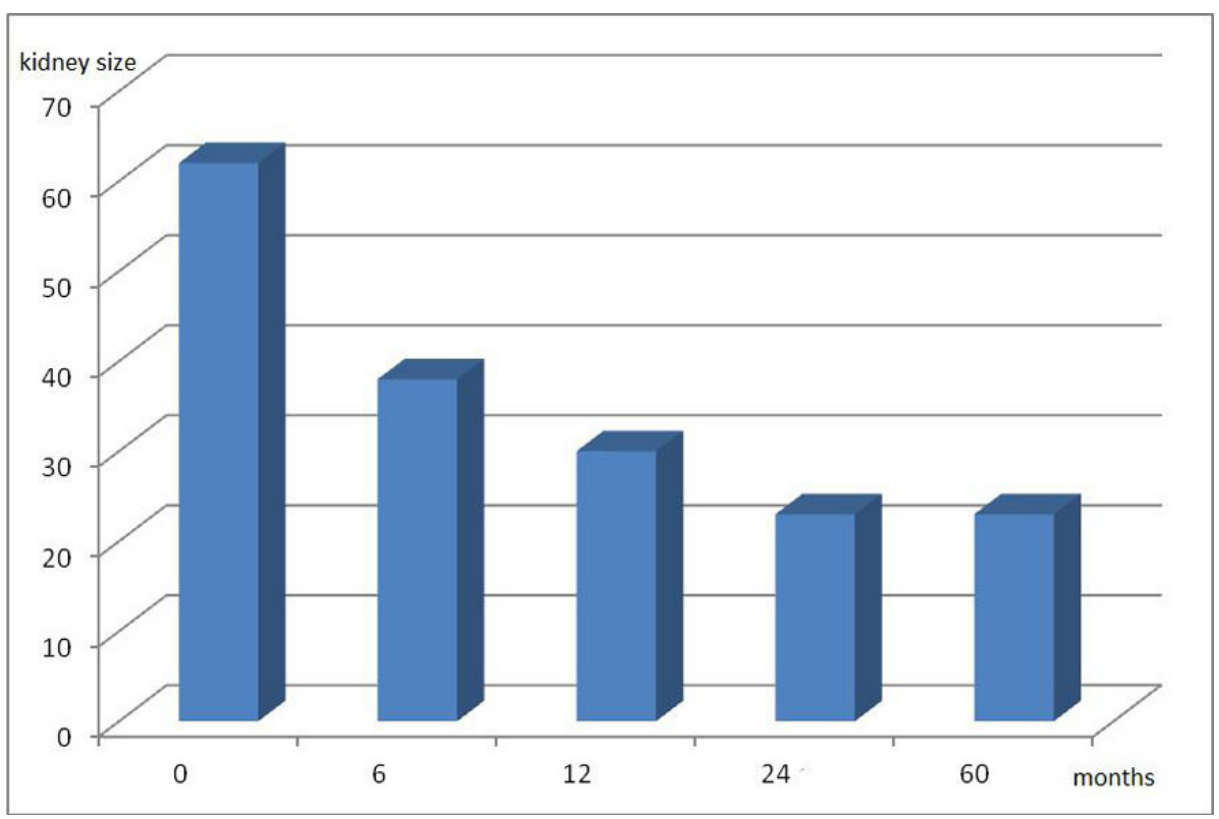

Figure 2. Evolution of GFR values $\left(\mathrm{mL} / \mathrm{min} / 1.73 \mathrm{~m}^{2}\right)$ in children with renal dysplasia and hypoplasia. 
and posteriourethral valve in 1 . Three of patients with VUR $(50 \%)$ resolved spontaneously during follow-up, $2(25 \%)$ required endoscopic treatment by subureteric injection of a bulking agent, and only one (12.5\%) required ureteral reimplantation. Eleven patients $(22.9 \%)$ had compensatory hypertrophy in the contralateral kidney. Other associated congenital abnormalities included dilated cardiomyopathy in 2 patients, down syndrome in 1, hydrocele in 1, and cryptorchidism in 1.

During the follow-up, 21 patients (43.7\%) showed completely involution within 2 years and 16 (33.3\%) showed a partial involution on US scan. Kidney length remained almost unchanged in 11 children $(22.9 \%)$. The univariate analysis showed that no variables (gender, additional urological anomalies, kidney length, history of urinary tract infection, proteinuria, and hypertension) were associated with complete involution. Figure 1 shows the regression rate of dysplastic kidneys.

On laboratory evaluation, the mean creatinine level was $1.66 \pm 2.1 \mathrm{mg} / \mathrm{dL}$ and the mean estimated GFR (eGFR) was $75.8 \pm 55.6 \mathrm{~mL} / \mathrm{min}$ (Fig. 2). Eleven of 48 patients $(22.9 \%)$ had an eGFR $<30 \mathrm{~mL} / \mathrm{min} / 1.73 \mathrm{~m}^{2}$, of which 7 had contralateral renal dysplasia and 4 had contralateral reflux nephropathy. Also 14 patients $(29.1 \%)$ had evidence for hyperfiltration (eGFR $>130 \mathrm{~mL} / \mathrm{min} / 1.73 \mathrm{~m}^{2}$ ). Nevertheless, 7 children developed CRF during the follow-up and at last follow-up, 18 children were in end-stage renal failure $(37.5 \%)$.

Fifteen children $(31.2 \%)$ were diagnosed to have urinary tract infection (UTI) based on positive urine cultures and symptoms of UTI. Proteinuria was noted in 11 (22.9\%) patients, of whom all had advanced CRF with nephrotic range proteinuria. Fifteen $(31.2 \%)$ children had hypertension at the diagnosis but no children developed new onset hypertension during the follow-up. Furthermore, no neoplastic degeneration was documented in any case.

\section{Discussion}

The prevalence of dysplastic kidneys has increased in the last two decades because of the widespread use of prenatal and postnatal US [10]. However, information on the natural course of these patients is still limited [5, 13-15]. We report here our center's experience on the outcome of dysplastic kidneys in a homogeneous pediatric population.

In our study, the rates of complete involution, partial involution, and unchanged size were $43 \%, 33 \%$, and $23 \%$, respectively. Complete involution occurred within the first 2 years in all patients. Various reports have documented a complete involution rate of $0 \%$ to $74 \%$ over 9 months to 10 years [13-16]. Our results were consistent with those reported previously. We also found that no variables (gender, additional urological anomalies, kidney length, history of urinary tract infection, proteinuria, and hypertension) were associated with complete involution.

In this study, the management was conservative in all cases and there was no malignant transformation to Wilms' tumor or renal cell carcinoma over a follow-up period of 60 months. In 1997, Beckwith reproted that the risk of Wilms' tumor is 1 in 10,000 in the general pediatric population and $3-10$ in 10,000 in children with dysplastic kidney [17]. In a review, Narchi collected all published cohort studies involving 1,041 children about outcome of dysplastic kidneys and he found no cases with Wilms' tumor or other malignancies [7]. Thus, we believe that elective nephrectomy of non-functioning dysplastic kidneys does not appear necessery unless there are other serious complications.

Children with renal dysplasia have an increased risk for congenital urogenital abnormalities with a prevalence varying from $10 \%$ to $50 \%$ [18-20]. In our study, 26 patients (54\%) had pathologic findings on the contralateral kidney including hydronephrosis in $35.4 \%$, VUR in $12.5 \%$, and renal dysplasia in $14.5 \%$ as the most common abnormalities. The reported incidence of reflux in patients with dysplastic kidneys varies widely; the present incidence was only $12.5 \%$ [10]. Three of them resolved spontaneously during followup, 2 required endoscopic subureteric injection, and one required ureteral reimplantation. Children with dysplastic kidney also have a higher risk of UTI. The risk of UTI appears to be assocaited with VUR or coexistent urological abnormalities rather than the dysplastic kidney. Thus children with VUR have a greater risk of pyelonephritis, renal damage, renal insufficiency, and hypertension, especially when the diagnosis of UTI is delayed.

Eleven patients $(22.9 \%)$ had CRF, of which 7 had contralateral renal dysplasia and 4 had contralateral reflux nephropathy. Nevertheless, 7 children developed CRF during the follow-up and at last follow-up 18 children $(37.5 \%)$ were in end-stage renal failure. Proteinuria, hypertension, and GFR are the variables commonly used for renal function monitoring [21]. In our study, 15 (31.2\%) children had hypertension at the diagnosis but no children developed new onset hypertension during the follow-up. We think that the main risk factor for developing hypertension is related to dysplastic end-stage kidneys or damage to the functional contralateral kidney.

\section{Conclusion}

Dysplastic kidneys are increasingly being detected on routine prenatal US. We report here the natural history of prenatally detected and conservatively managed children with dysplastic kidney. The prognosis of these patients depends on the function of the contralateral kidney. Thus, we recommend that patients should be monitored regularly assessing the dysplastic and contralateral kidney, blood pressure, urinalysis, and renal function. 


\section{Grant Support}

There is no any grant or financial support.

\section{References}

1. Fraser N, Paul A, Williams AR, Broderick N, Shenoy MU. Dysplastic kidneys in children - do they grow? J Pediatr Urol. 2010;6(1):40-44.

2. Hains DS, Bates CM, Ingraham S, Schwaderer AL. Management and etiology of the unilateral multicystic dysplastic kidney: a review. Pediatr Nephrol. 2009;24(2):233-241.

3. Ismaili K, Schurmans T, Wissing KM, Hall M, Van Aelst C, Janssen F. Early prognostic factors of infants with chronic renal failure caused by renal dysplasia. Pediatr Nephrol. 2001;16(3):260-264.

4. Kerecuk L, Schreuder MF, Woolf AS. Renal tract malformations: perspectives for nephrologists. Nat Clin Pract Nephrol. 2008;4(6):312-325.

5. Winyard P, Chitty LS. Dysplastic kidneys. Semin Fetal Neonatal Med. 2008;13(3):142-151.

6. Gonzalez Celedon C, Bitsori M, Tullus K. Progression of chronic renal failure in children with dysplastic kidneys. Pediatr Nephrol. 2007;22(7):1014-1020.

7. Narchi H. Risk of Wilms' tumour with multicystic kidney disease: a systematic review. Arch Dis Child. 2005;90(2):147-149.

8. Narchi H. Risk of hypertension with multicystic kidney disease: a systematic review. Arch Dis Child. 2005;90(9):921-924.

9. Dinkel E, Ertel M, Dittrich M, Peters H, Berres M, Schulte-Wissermann H. Kidney size in childhood. Sonographical growth charts for kidney length and volume. Pediatr Radiol. 1985;15(1):38-43.

10. Kuwertz-Broeking E, Brinkmann OA, Von Lengerke HJ, Sciuk J, Fruend S, Bulla M, Harms E, et al. Unilateral multicystic dysplastic kidney: experience in children. BJU Int. 2004;93(3):388-392.
11. Blumenthal S, Epps RP, Heavenrich R, Lauer RM, Lieberman E, Mirkin B, Mitchell SC, et al. Report of the task force on blood pressure control in children. Pediatrics. 1977;59(5 2 suppl):I-II, 797-820.

12. Schwartz GJ, Haycock GB, Spitzer A. Plasma creatinine and urea concentration in children: normal values for age and sex. J Pediatr. 1976;88(5):828-830.

13. Mattioli G, Pini-Prato A, Costanzo S, Avanzini S, Rossi V, Basile A, Ghiggeri GM, et al. Nephrectomy for multicystic dysplastic kidney and renal hypodysplasia in children: where do we stand? Pediatr Surg Int. 2010;26(5):523-528.

14. Mansoor O, Chandar J, Rodriguez MM, Abitbol CL, Seeherunvong W, Freundlich M, Zilleruelo G. Long-term risk of chronic kidney disease in unilateral multicystic dysplastic kidney. Pediatr Nephrol. 2011;26(4):597-603.

15. Aslam M, Watson AR. Unilateral multicystic dysplastic kidney: long term outcomes. Arch Dis Child. 2006;91(10):820-823.

16. Onal B, Kogan BA. Natural history of patients with multicystic dysplastic kidney-what followup is needed? J Urol. 2006;176(4 Pt 1):1607-1611.

17. Homsy YL, Anderson JH, Oudjhane K, Russo P. Wilms tumor and multicystic dysplastic kidney disease. J Urol. 1997;158(6):2256-2259; discussion 2259-2260.

18. Damen-Elias HA, Stoutenbeek PH, Visser GH, Nikkels PG, de Jong TP. Concomitant anomalies in 100 children with unilateral multicystic kidney. Ultrasound Obstet Gynecol. 2005;25(4):384-388.

19. Belk RA, Thomas DF, Mueller RF, Godbole P, Markham AF, Weston MJ. A family study and the natural history of prenatally detected unilateral multicystic dysplastic kidney. J Urol. 2002;167(2 Pt 1):666-669.

20. Merrot T, Lumenta DB, Tercier S, Morisson-Lacombes G, Guys JM, Alessandrini P. Multicystic dysplastic kidney with ipsilateral abnormalities of genitourinary tract: experience in children. Urology. 2006;67(3):603-607.

21. Caione P, Villa M, Capozza N, De Gennaro M, Rizzoni G. Predictive risk factors for chronic renal failure in primary high-grade vesico-ureteric reflux. BJU Int. 2004;93(9):1309-1312. 